\title{
Endoscopic management of maxillary sinus inverted papilloma attachment sites to minimize disease recurrence
}

\author{
Vincent $\mathrm{Wu}^{1}$, Jennifer $\mathrm{Siu}^{2}$, Jonathan Yip ${ }^{2}$ and John M. Lee $2,3^{*}$
}

\begin{abstract}
Background: Inverted papillomas (IPs) are benign neoplasms, most commonly arising from the mucosal lining of the maxillary sinus. IPs can have single or multifocal sites of attachment. Although pedicle location is an important factor to consider in surgical planning, it is less clear whether the location or number of IP attachment sites hold any prognostic value. Herein, we aimed to determine the prognostic significance of the number and location of attachment sites of IPs originating from the maxillary sinus when managed by a pure endoscopic approach.

Methods: This was a single-center, single-surgeon retrospective chart review. Patients with maxillary sinus IPs who were managed by endoscopic approaches only, from January 1, 2010 to June 30, 2016, were identified. Demographic data, operative technique, number and location of IP attachment sites, follow-up duration, recurrence, and presence of malignant transformation were captured.

Results: Twenty-eight maxillary IP patients (61\% males) were included, with a mean age of 54.9 (standard deviation (SD): 16.5$)$ years. Approximately $36 \%$ of patients were referred from other institutions for management of recurrent IPs after failing previous surgical treatment. All patients were managed with an endoscopic approach, and all required an endoscopic medial maxillectomy to facilitate access to the maxillary sinus. At a mean follow-up of 31.1 (SD: 22.6) months, there were no recurrences identified. IPs with single (46\%) and multifocal (54\%) attachments were predominately to the medial and lateral walls. Maxillary IPs with multifocal attachments most frequently involved 2-3 walls of the sinus. Osteitis (36\%) was commonly seen.

Conclusion: IPs originating from the maxillary sinus frequently had multifocal attachments, but this did not impact disease recurrence. Despite the surgical challenges of accessing all of the maxillary sinus walls, IPs originating from the maxillary sinus can be effectively managed via a pure endoscopic approach.
\end{abstract}

Keywords: Endoscopic, Sinus surgery, Maxillary sinus, Inverted papilloma, Prognosis, Recurrence, Attachment site, Multifocal, Medial maxillectomy

\section{Background}

Inverted papillomas (IPs) are benign neoplasms arising from the mucosal lining of the nasal cavity and paranasal sinuses [1]. IPs can have either single or multifocal sites of origin, with a recurrence rate ranging from 14 to $25 \%$ if surgical resection is not

\footnotetext{
*Correspondence: leejo@smh.ca

${ }^{2}$ Division of Rhinology, Department of Otolaryngology - Head and Neck

Surgery, St. Michael's Hospital, University of Toronto, Toronto, Ontario, Canada

${ }^{3}$ Li Ka Shing Knowledge Institute, St. Michael's Hospital, Toronto, Ontario,

Canada

Full list of author information is available at the end of the article
}

complete [2, 3]. This is of clinical significance as IPs are associated with a $5-15 \%$ malignant transformation rate to squamous cell carcinoma $[4,5]$.

The majority of IPs develop within the maxillary sinus, often originating from the medial wall [6-8]. Invasion into adjacent structures is the main complication of disease progression and can involve the orbit, lacrimal system, and skull base [9-12]. These tumors also have a tendency to erode and re-model bone, leading to devastating sequelae $[13,14]$.

Surgery is the mainstay of treatment for IPs and historically have included several approaches: 1) non-endoscopic 
endonasal, 2) limited external (i.e. Caldwell-Luc), 3) radical external (i.e. lateral rhinotomy or midfacial degloving with en bloc resection), and 4) endoscopic endonasal [5, 7, 12, 15]. A pedicle-oriented strategy is currently widely implemented in the resection of IPs with the surgical approach employed specific to the pedicle location. It has been reported that the endoscopic approach alone was insufficient in reaching all maxillary IPs pedicle sites, especially with IPs that originated from the lateral, anterior, and inferior sinus walls [7]. In these instances, external approaches such as the Caldwell-Luc were required for complete resection [7]. However, with advancements in endoscopic technologies and techniques, tumors originating from the maxillary sinus have increasingly been managed by a pure endoscopic approach alone.

Although the pedicle location is an important factor to consider in surgical planning, it is less clear whether the location or number of IP attachment sites hold any prognostic value. Herein, we aimed to determine the prognostic significance of the number and location of attachment sites of IPs originating from the maxillary sinus, when managed by a pure endoscopic approach. We hypothesize that IPs of multiple attachment sites are associated with increased recurrence.

\section{Methods}

\section{Patient selection}

We carried out a single surgeon (JML), single centre retrospective chart review. All patients who had resection for IPs originating only from the maxillary sinus, from January 1, 2010 to June 30, 2016, were included in the study. Patients who had expressed prior wishes not to be included in any clinical research at our academic centre were excluded. There were no other exclusion criteria.

Patient charts including pre-operative consults, operative notes, and post-operative follow-up reports were obtained and reviewed. All operative notes were dictated by the staff surgeon (JML). Demographic data, history of previous IP resection, surgical technique(s), presence of any perioperative complication(s), length of stay (LOS), and follow-up length were extracted.

\section{Surgical approach}

Once pathology of IP was confirmed via intraoperative frozen sections, the mass was resected systematically with a microdebrider initially to debulk the tumor that was free-floating in the sinonasal cavity. The goal of the surgery is to identify the site(s) of attachment(s) so that the mucosa can be removed and the underlying bone drilled down to decrease the chance of tumor recurrence. Angled endoscopes, along with curved instruments, microdebriders and burrs were used for tumor resection within the maxillary sinus. A medial maxillectomy was performed to facilitate removal of the tumor origin (if it was attached to the medial maxillary sinus wall) or simply to allow increased access to all walls of the sinus cavity. For IPs originating from the anterior maxillary wall, a transseptal approach was used for increased angulation [16]. Frozen sections of resection margins were sent at the end of the operation to ensure complete removal of IP.

\section{Outcome measures}

The primary outcome measures were IP attachment site(s) and recurrence. The number and location(s) of the IP attachment site(s) were determined based on direct visualization by the surgeon, and were extracted from the operative note. A secondary measure was the presence of malignant transformation including dysplasia.

\section{Statistical analysis}

Descriptive statistics were used to summarize the frequency and percentage of categorical variables. Continuous variables are reported as mean and standard deviation (SD). Student's t-test was performed to determine the differences between single and multiple sites of IP attachment for age, follow-up time, LOS, and disease recurrence. Fisher's exact test was used to analyze differences in gender and previous recurrence. All statistical analyses were performed using Prism (v.7, GraphPad, USA), with significance set to $\alpha=0.05$.

\section{Results}

Twenty-eight maxillary IP patients (61\% males) were identified and included for analysis, with a mean age of 54.9 (SD: 16.5) years. At a mean follow-up of 31.1 (SD: 22.6) months, no recurrences were identified. Ten patients (36\%) were referred for IPs, which recurred after failing initial surgical treatment from another surgeon. Osteitis (36\%) was commonly seen. Patient characteristics are shown in Table 1.

Comparisons of baseline characteristics between single and multiple attachment sites IPs are shown in Table 2 . There was no statistically significant difference in the rate of recurrence between single and multiple attachment IPs

Table 1 Baseline Characteristics

\begin{tabular}{ll}
\hline Variables & Total Patient $(n=28)$ \\
\hline Gender (male:female) & $17: 11$ \\
Age, years (mean, SD) & $54.9(16.5)$ \\
Prev. recurrence $(n, \%)$ & $10(36 \%)$ \\
Recurrence $(n, \%)$ & $0(0.0 \%)$ \\
Malignant transformation (,$\%)$ & $0(0.0 \%)$ \\
Follow-up, months (mean, SD) & $31.1(22.6)$ \\
Length of stay, days (mean, SD) & $0.68(1.3)$ \\
Perioperative complications ( $n, \%)$ & $0(0.0 \%)$ \\
\hline
\end{tabular}


Table 2 Comparison of Baseline Characteristics between Single and Multiple Attachment Sites

\begin{tabular}{llll}
\hline Variables & Single Attach. $(n=13)$ & Multiple Attach. $(n=15)$ & $P$-value \\
\hline Gender (male:female) & $7: 6$ & $10: 5$ & 0.700 \\
Age, years (mean, SD) & $55.8(16.8)$ & $54.2(16.8)$ & 0.807 \\
Prev. recurrence $(n, \%)$ & $3(23 \%)$ & $7(47 \%)$ & 0.254 \\
Follow-up, months (mean, SD) & $37.6(23.7)$ & $25.4(20.7)$ & 0.158 \\
Length of stay, days (mean, SD) & $0.31(0.48)$ & $1.0(1.6)$ & 0.145 \\
\hline
\end{tabular}

group prior to revision surgery. Additionally, no statistically significant difference was noted in follow-up time between single attachment IP patients, with a length of 37.6 (SD: 23. 7) months, as compared to 25.4 (SD: 20.7) months in multifocal IP patients. LOS in hospital was short within both groups, and did not differ between groups.

Attachments of IP to the maxillary sinus walls were classified as either single (46\%) or multiple (54\%). IPs with single attachment predominately originated from the medial maxillary wall, while those with multiple pedicles involved primarily the medial, lateral, and anterior walls (Table 3). For maxillary IPs with multifocal sites of attachment, $67 \%$ of cases originated from 2 to 3 walls of the sinus cavity, but can also have multiple attachments to a single wall (Table 4).

All patients were managed with a pure endoscopic approach, with all requiring an endoscopic medial maxillectomy. No adjunctive external approaches were required and no perioperative complications were encountered.

\section{Discusssion}

The complete resection of maxillary sinus IPs is crucial due to the propensity for recurrence and its malignancy risk. During surgery, the tumor must be followed down to its origin to facilitate a wide local excision of the pedicle site $[7,17,18]$. In our series, IPs were followed to their attachments, with diseased mucosa resected and bone drilling performed at the pedicle sites. The number of attachment sites can theoretically be associated with tumor recurrence, since most IP recurrences occur at the pedicle and multifocal IPs may be more difficult to manage surgically $[7,19]$. Previous reports have demonstrated successful surgical results stemming from a pure endoscopic approach for maxillary IPs originating from

Table 3 Wall of Maxillary Sinus Involved in Cases with Single and Multiple Attachment Sites

\begin{tabular}{lll}
\hline Maxillary Sinus Wall & Single Attach. & Multiple Attach. \\
\hline Posterior & 1 & 7 \\
Inferior & 0 & 7 \\
Lateral & 2 & 9 \\
Anterior & 1 & 9 \\
Medial & 8 & 10 \\
Superior & 1 & 3 \\
\hline
\end{tabular}

the medial, superior, and posterior walls $[4,7,20,21]$. However, endoscopic access to the anterior and inferior walls was noted to be more challenging, inadequate for reaching all of the tumor, which often necessitated adjunctive external approaches [7, 21-23].

Dean et al., (2014) reported that using a transseptal surgical approach in combination with medial maxillectomy facilitated full visualization of anterolateral maxillary IPs with multiple attachment sites, which allowed for the complete resection of the tumor pedicle [16]. During their study, with a mean follow-up of 29 months, no recurrences were noted for both the single and multiple IP attachment site groups [16]. Compared to results from our study, this is a similar findings as we noted no disease recurrence between single and multifocal sites at 31.1 months, while using a similar transseptal approach for accessing the anterior maxillary wall. However, contrasting our findings of no perioperative complications, Dean et al. reported infraorbital paresthesia in $9 \%$ of patients, secondary to tumor invasion of the nerve [16].

In a study by Hong et al. (2015), evaluating surgical approaches used in resecting maxillary IPs, only $16.1 \%$ of patients underwent a pure endoscopic approach [7]. External approaches, including Canine fossa puncture via Caldwell-Luc approach or Caldwell-Luc operation, were added gradually as required if endoscopy was insufficient in resecting all parts of the IP. External approaches were mainly added for lateral, anterior, and inferior wall involvement [7]. Hong et al. showed that $48.4 \%$ of maxillary IPs originated from the anterior and/or inferior walls, comparable to $42.9 \%$ in our study [7]. Similar to our results, no recurrences were reported in patients who underwent a pure endoscopic approach at a mean followup of 64.2 months [ 7]. Of interest, the recurrence rate

Table 4 Number of Maxillary Sinus Walls Involved in Cases of Multifocal Attachment

\begin{tabular}{ll}
\hline Number of Walls Involved & Cases \\
\hline 1 & 2 \\
2 & 5 \\
3 & 5 \\
4 & 1 \\
5 & 0 \\
6 & 2 \\
\hline
\end{tabular}


was found to be $9.7 \%$ in patients with IPs of multiple attachment sites, who underwent adjunctive external approaches [7]. In our experience, we found that an external approach is not necessary for access to all IP attachment sites. Instead, an endoscopic medial maxillectomy and use of angled scopes can facilitate visualization to the anterior and inferior maxillary walls.

The results of our study have several clinical implications. First, pure endoscopic management of sino-nasal disease decreases the need for prolonged LOS in hospital. A previous report by Sautter et al. (2007) demonstrated that patients who underwent endoscopic IP resection spent significantly fewer days in hospital as compared to patients who had open surgery [24]. Similarly, our study demonstrates low LOS of both our single and multifocal IPs. Other clinical implications of a pure endoscopic approach to maxillary sinus IPs have been well described in the literature and include avoidance of facial incision, minimizing scarring, decreased pain, swelling, and dysesthesia as compared to open surgery [20, 22]. Moreover, one of the largest single cohort studies on IP reported a reduction in recurrence with the implementation of endoscopic approaches [25]. These results were reiterated by a recent meta-analysis, which noted that endoscopic management of IPs reduced recurrence risk by $44 \%$ as compared to external approaches [26]. Additionally, endoscopic medial maxillectomy has been described as effective and reproducible for IP resection, with decreased operating time and morbidity as compared to open maxillectomy [27]. As we noted no perioperative complications within our series, our results may provide further evidence indicating the low morbidity stemming from the endoscopic approach.

This study has limitations. While we aimed to acquire long-term data on IP recurrence within our cohort, with a mean follow-up of 31.1 months, this still may not have adequately captured all cases of recurrence. It has been reported that up to $20 \%$ of recurrences may occur 5 years after resection for all IPs [28]. However, specific for maxillary IPs, recurrences have been noted to occur within a mean time of 20 months [7]. This study was also retrospective in nature, and has limitations inherent to such analysis. Our analysis focused on surgical outcomes by distinguishing single from multiple attachment sites IPs. With a larger sample size, analysis may be performed for surgical outcomes based on the exact number of attachments. Future studies should aim to assess longerterm outcomes.

\section{Conclusion}

No differences in recurrence were noted between single and multifocal attachment maxillary IPs. The majority of IPs originating from the maxillary sinus frequently had multi-focal attachments. Despite surgical challenges of reaching all sinus walls, maxillary IPs may be managed effectively via a pure endoscopic approach.

\section{Abbreviations}

IP: Inverted papilloma; LOS: Length of stay; SD: Standard deviation

\section{Acknowledgements}

This study was presented as a poster presentation at the Canadian Society of Otolaryngology - Head and Neck Surgery annual meeting in Saskatoon, Saskatchewan, 2017.

Funding

This study received no funding.

Availability of data and materials

The datasets generated and analysed during the current study are available from the corresponding author on reasonable request.

\section{Authors' contributions}

All authors were involved with the conception and design of the study, analysis and interpretation of data, revision of the manuscript, and have approved the final manuscript.

Ethics approval and consent to participate

The St. Michael's Hospital Research Ethics Board approved this study.

Consent for publication

Not applicable.

Competing interests

The authors declare that they have no competing interests.

\section{Publisher's Note}

Springer Nature remains neutral with regard to jurisdictional claims in published maps and institutional affiliations.

\section{Author details}

${ }^{1}$ School of Medicine, Faculty of Health Sciences, Queen's University, Kingston, Ontario, Canada. ${ }^{2}$ Division of Rhinology, Department of Otolaryngology -

Head and Neck Surgery, St. Michael's Hospital, University of Toronto, Toronto, Ontario, Canada. ${ }^{3}$ Li Ka Shing Knowledge Institute, St. Michael's Hospital,

Toronto, Ontario, Canada.

Received: 6 February 2018 Accepted: 27 March 2018

Published online: 04 April 2018

\section{References}

1. Wood JW, Casiano RR. Inverted papillomas and benign nonneoplastic lesions of the nasal cavity. Am J Rhinol Allergy. 2012;26(2):157.

2. Shahrjerdi B, AngoYaroko A, Abdullah B. Co-existing of sinonasal inverted papilloma and angiofibroma: care report and review of the literature. Acta Inform Med. 2012;20(4):261-3.

3. Mirza S, Bradley PJ, Acharya A, Stacey M, Jones NS. Sinonasal inverted papillomas: recurrence, and synchronous and metachronous malignancy. J Laryngol Otol. 2007;121(9):857-64.

4. Krouse $\mathrm{JH}$. Endoscopic treatment of inverted papilloma: safety and efficacy. Am J Otolaryngol. 2001;22(2):87-99.

5. Hyams VJ. Papillomas of the nasal cavity and the paranasal sinuses, a clinicopathologic study of 315 cases. Ann Otol Rhinol Laryngol. 1971; 80(2):192-6

6. Buchwald C, Nielsen LH, Nielsen PL, Ahlgren P, Tos M. Inverted papilloma: a follow-up study including primarily unacknowledged cases. Am J Otolaryngol. 1989;10(4):273-81.

7. Hong SL, Mun SJ, Cho KS, Roh HJ. Inverted papilloma of the maxillary sinus: surgical approach and long-term results. Am J Rhinol Allergy. 2015;29(6):441-4.

8. Schneyer MS, Milam BM, Payne SC. Sites of attachment of Schneiderian papilloma: a retrospective analysis. Int Forum Allergy Rhinol. 2011;1(4):324-8.

9. Chaudhry IA, Taiba K, Al-Sadhan Y, Riley FC. Inverted papilloma invading the orbit through the nasolacrimal duct: a case report. Orbit. 2005;24(2):135-9. 
10. Bajaj MS, Pushker N. Inverted papilloma invading the orbit. Orbit. 2002; 21(2):155-9.

11. Elner VM, Burnstine MA, Goodman ML, Dortzbach RK. Inverted papillomas that invade the orbit. Arch Ophthalmol. 1995;113(9):1178-83.

12. Pitak-Arnnop P, Bertolini J, Dhanuthai K, Hendricks J, Hemprich A, Pausch NC. Intracranial extension of Schneiderian inverted papilloma: a case report and literature review. Ger Med Sci. 2012;10:12.

13. Krouse $\mathrm{JH}$. Development of a staging system for inverted papilloma. Laryngoscope. 2000;110(6):965-8.

14. Wolfe SG, Schlosser RJ, Bolger WE, Lanza DC, Kennedy DW. Endoscopic and endoscope-assisted resections of inverted sinonasal papillomas. Otolaryngol Head Neck Surg. 2004;131:174-9.

15. Sham CL, Woo JK, van Hasselt CA. Endoscopic resection of inverted papilloma of the nose and paranasal sinuses. J Laryngol Otol. 1998; 112(8):758-64

16. Dean NR, Illing EA, Woodworth BA. Endoscopic resection of anterolateral maxillary sinus inverted papillomas. Laryngoscope. 2015;125(4):807-12

17. Xiao-Ting W, Peng L, Xiu-Qing W, Hai-Bo W, Wen-Hui P, Bing L, Er-Peng Z, Guang-Gang S. Factors affecting recurrence of sinonasal inverted papilloma. Eur Arch Otorhinolaryngol. 2013:1-5.

18. Kamel RH, Abdel FA, Awad AG. Origin oriented management of inverted papilloma of the frontal sinus. Rhinology. 2012;50(3):262-8.

19. Dubin MG, Kuhn FA. Unilateral multifocal inverted papilloma of the maxillary and frontal sinus. Am J Otolanyngol. 2006;27(4):263-5.

20. Lund V, Stammberger H, Nicolai P, Castelnuovo P. European position paper on endoscopic Management of Tumours of the nose, paranasal sinuses and Skull Base introduction. Rhinology. 2011:1-43.

21. Dragonetti A, Gera R, Sciuto A, Scotti A, Bigoni A, Barbaro E, Minni A. Sinonasal inverted papilloma: 84 patients treated by endoscopy and proposal for a new classification. Rhinology. 2011;49(2):207-13.

22. Busquets JM, Hwang PH. Endoscopic resection of sinonasal inverted papilloma: a meta-analysis. Otolaryngol Head Neck Surg. 2006;134(3):476-82.

23. McCollister KB, Hopper BD, Ginsberg LE, Michel MA. Inverted papilloma: a review and What's new. Neurographics. 2015;5(3):96-103.

24. Sautter NB, Cannady SB, Citardi MJ, Roh HJ, Batra PS. Comparison of open versus endoscopic resection of inverted papilloma. Am J Rhinol. 2007;21(3):320-3.

25. Bugter O, Monserez DA, van Zijl FVWJ, Baatenburg de Jong RJ, Hardillo JA. Surgical management of inverted papilloma; a single-center analysis of 247 patients with long follow-up. J Otolaryngol Head Neck Surg. 2017 Dec 20;46(1):67.

26. Amedee RG. Recurrence of Sinonasal inverted papilloma following surgical approach: a meta-analysis. Am J Rhinol. 2017;31(3):207.

27. Sadeghi N, Al-Dhahri S, Manoukian JJ. Transnasal endoscopic medial maxillectomy for inverting papilloma. Laryngoscope. 2003;113(4):749-53.

28. Jiang XD, Dong QZ, Li SL, Huang TQ, Zhang NK. Endoscopic surgery of a sinonasal inverted papilloma: surgical strategy, follow-up, and recurrence rate. Am J Rhinol Allergy. 2017;31(1):51-5.

\section{Submit your next manuscript to BioMed Central and we will help you at every step:}

- We accept pre-submission inquiries

- Our selector tool helps you to find the most relevant journal

- We provide round the clock customer support

- Convenient online submission

- Thorough peer review

- Inclusion in PubMed and all major indexing services

- Maximum visibility for your research

Submit your manuscript at www.biomedcentral.com/submit

CBiomed Central 